\title{
A CHARACTERIZATION OF $F^{+} \cap N$
}

\author{
M. STOLL
}

\begin{abstract}
In this note we give a characterization of $F^{+} \cap N$, where $N$ denotes the Nevanlinna class of functions of bounded characteristic and $F^{+}$ denotes the containing Fréchet space of $N^{+}$. We show that a holomorphic function $f \in F^{+} \cap N$ if and only if $f(z)=g(z) / S_{\mu}(z)$, where $g \in N^{+}$and $S_{\mu}$ is a singular inner function with respect to a nonnegative continuous singular measure $\mu$.
\end{abstract}

Let $U$ denote the unit disc $\{|z|<1\}$ and let $T$ denote the boundary of $U$. As in [1], we denote by $N$ the Nevanlinna class of functions of bounded characteristic on $U$ and by $N^{+}$the subspace of $N$ consisting of those functions $f \in N$ for which

$$
\lim _{r \rightarrow 1^{-}} \frac{1}{2 \pi} \int_{0}^{2 \pi} \log ^{+}\left|f\left(r e^{i t}\right)\right| d t=\frac{1}{2 \pi} \int_{0}^{2 \pi} \log ^{+}\left|f\left(e^{i t}\right)\right| d t,
$$

where $f\left(e^{i t}\right)=\lim _{r \rightarrow 1^{-}} f\left(r e^{i t}\right)$ a.e. on $T$. The space $N^{+}$with the metric $\rho$ given by

$$
\rho(f, g)=\lim _{r \rightarrow 1^{-}} \frac{1}{2 \pi} \int_{0}^{2 \pi} \log \left(1+\left|f\left(r e^{i t}\right)-g\left(r e^{i t}\right)\right|\right) d t
$$

is an $F$-space [5]. The space $F^{+}$, the containing Fréchet space of $N^{+}$, consists of those functions $f$ holomorphic in $U$ satisfying

$$
\limsup _{r \rightarrow 1^{-}}(1-r) \log M(r, f) \leqslant 0
$$

where $M(r, f)=\max _{|z|=r}|f(z)|[6]$.

Every function $f \in N$ can be factored uniquely as follows [1, p. 25]:

$$
f(z)=B(z)\left(S_{\mu_{1}}(z) / S_{\mu_{2}}(z)\right) F(z),
$$

where $B(z)$ is the Blaschke product with respect to the zeros of $f(z), F(z)$ is an outer function, and $S_{\mu_{1}}(z)$ and $S_{\mu_{2}}(z)$ are singular inner functions with respect to the nonnegative singular measures $\mu_{1}$ and $\mu_{2}$ and are given by

$$
S_{\mu_{j}}(z)=\exp \left[-\int_{0}^{2 \pi} \frac{e^{i t}+z}{e^{i t}-z} d \mu_{j}(t)\right], \quad j=1,2 .
$$

A function $f(z) \in N$ is in $N^{+}$if and only if $S_{\mu_{2}}(z) \equiv 1$, i.e., $\mu_{2}=0$.

In [7], N. Yanagihara has constructed an example of a function $f \in F^{+}$

Received by the editors January 7, 1975 and, in revised form, April 21, 1975.

AMS (MOS) subject classifications (1970). Primary 30A76, 31A20.

- American Mathematical Society 1976 
$\cap N$ but not in $N^{+}$. In this note, using some results of [3], [4] we obtain the following characterization of $F^{+} \cap N$.

THEOREM. $F^{+} \cap N=\left\{f / S_{\mu}: f \in N^{+}, \mu\right.$ a nonnegative continuous singular measure $\}$, where $S_{\mu}$ is given by (4).

Proof. Recall that a measure $\mu$ on $T$ is continuous if and only if $\mu(\{\omega\})=0$ for all $\omega \in T$. As in [4], for each $\omega \in T$, we define $\lambda_{\omega}$ on $N$ by

$$
\lambda_{\omega}(f)=\limsup _{r \rightarrow 1^{-}}(1-r) \log ^{+}|f(r \omega)| \text {. }
$$

By (3) every $f \in N$ can be written as $f(z)=g(z) / S_{\mu}(z)$, where $g \in N^{+}, S_{\mu}$ is a singular inner function given by (4), and the singular inner function in the factorization (3) of $g$ and $S_{\mu}$ has no common divisor. By Theorem (2.2) of [4], if $f \in N$ and $\omega \in T, \lambda_{\omega}(f)=2 \mu(\{\omega\})$. Consequently, if $f \in F^{+} \cap N$, by (2), $\lambda_{\omega}(f)=0$ for all $\omega \in T$ and hence by the above, $\mu(\{\omega\})=0$ for all $\omega \in T$, i.e., $\mu$ is continuous.

Conversely, suppose $f=g / S_{\mu}$, where $g \in N^{+}$, and $\mu$ is a nonnegative continuous singular measure. Clearly $f \in N$, and since $F^{+}$is closed under multiplication, it suffices to show that $1 / S_{\mu} \in F^{+}$. By the remark on p. 164 of [3],

$$
\left|S_{\mu}\left(r e^{i t}\right)\right|>\exp [-C \omega(1-r) /(1-r)]
$$

where $\omega(h)$ is the modulus of continuity of the distribution function of $\mu$, and $C$ is a positive constant. Consequently, since $\mu$ is continuous,

$$
\limsup _{r \rightarrow 1^{-}}(1-r) \log M\left(r, 1 / S_{\mu}\right) \leqslant 0 .
$$

Therefore by (2), $1 / S_{\mu} \in F^{+}$, which proves the result.

RemarK. In [4], J. H. Shapiro and A. L. Shields have shown that the space $N$ with the metric given by (1) is disconnected and they posed the problem of determining the component of the origin in $N$. This question has been answered by J. W. Roberts [2] where he has shown, using the above characterization of $F^{+} \cap N$, that the component of the origin in $N$ is $F^{+} \cap N$.

\section{REFERENCES}

1. P. L. Duren, Theory of $H^{p}$ spaces, Pure and Appl. Math., vol. 38, Academic Press, New York and London, 1970. MR 42 \#3552.

2. J. W. Roberts, The component of the origin in the Nevanlinna class, Illinois J. Math. 19 (1975), 553-559.

3. H. S. Shapiro, Weakly invertible elements in certain function spaces, and generators in $\ell_{1}$, Michigan Math. J. 11 (1964), 161-165. MR 29 \#3620.

4. J. H. Shapiro and A. L. Shields, Unusual topological properties of the Nevanlinna class (to appear).

5. N. Yanagihara, Multipliers and linear functionals for the class $N^{+}$, Trans. Amer. Math. Soc. 180 (1973), 449-461. MR 49 \#3147.

6. — The containing Fréchet space for the class $N^{+}$, Duke Math. J. 40 (1973), 93-103.

7. The class $\mathrm{N}^{+}$of holomorphic functions and its containing Fréchet space $\mathrm{F}^{+}$, Boll. Un. Mat. Ital. (4) 8 (1973), 230-245. MR 48 \#11520.

Department of Mathematics and Computer Science, University of South Carolina, Columbia, South Carolina 29208 